\title{
Comparison of Feedforward Network and Radial Basis Function to Detect Leukemia
}

\author{
Pragya Bagwari ${ }^{1}$, Bhavya Saxena, Meenu Balodhi², Vishwanath Bijalwan ${ }^{1} *$
}

${ }^{1}$ Gopeshwar (Uttarakhand Government Institute), Uttarakhand (India)

${ }^{2}$ Uttaranchal University Dehradun,Uttarakhand (India)

Received 3 September 2016 | Accepted 1 November 2016 | Published 23 December 2016

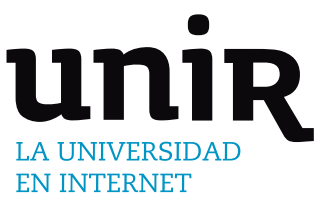

Leukemia is a fast growing cancer also called as blood cancer. It normally originates near bone marrow. The need for automatic leukemia detection system rises ever since the existing working methods include laborintensive inspection of the blood marking as the initial step in the direction of diagnosis. This is very time consuming and also the correctness of the technique rest on the worker's capability. This paper describes few image segmentation and feature extraction methods used for leukemia detection. Analyzing through images is very important as from images; diseases can be detected and diagnosed at earlier stage. From there, further actions like controlling, monitoring and prevention of diseases can be done. Images are used as they are cheap and do not require expensive testing and lab equipment. The system will focus on white blood cells disease, leukemia. Changes in features will be used as a classifier input.

K-mean Clustering, Texture Features, Feed Forward and RBFNN.

DOI: $10.9781 /$ ijimai.2017.4510

\section{INTRODUCTION}

$\mathrm{L}$ EUKEMIA is one of the many types of cancers. Leukemia is caused in the white blood cells near the bone marrow region of our body. In this the white blood cells (WBCs) which get infected turns blue. Like any other cancer in this also the cell divides itself at the faster pace. Even when it is not required they multiply causing a tumor. Detected and treated at an early stage of leukemia saves a lot of lives. According to leukemia research foundation, every four minutes someone is diagnosed with leukemia. More than 176,000 are expected in U.S.

Leukemia helps in detecting blood cancer using two basic modules of image processing i.e. Image segmentation and feature extraction. After these two modules we use two techniques of neural network i.e. feed forward network and radial basis function network (RBFNN) for the detection purposes. We compare the accuracy percentage in both of them. The technique with best accuracy percentage is recorded as the more efficient technique.

\section{RELATED Work}

Athira Krishnan, Sreekumar K [2] prposed a system to detect acute myelogenous leukemia which is a type of blood cancer. In this they have used two modules i.e. segmentation and feature extraction. In feature extraction they have extracted features like fractal dimensions, local binary patterns, texture features, shape and color features. Subrajeet Mohapatra, Dipti Patra et al [5] proposed a system unsupervised microscopic blood images using the data clustering techniques . In feature extraction shape and size of the cell. Ruggero Donida Labati IEEE Member, Vincenzo Piuri IEEE Fellow et al [8] they proposed an

* Corresponding author.

E-mail addresses: vishwanath.bijalwan@gmail.com open database for the new researchers so that they can avail the images easily. The images are segmented using basic techniques.

\section{METHODS}

The procedure for leukemia detection in the microscopic images consist of image segmentation and feature extraction. The working procedure is shown in the figure below (fig. 1). The leukemia consists of red blood cells (RBCs), WBCs and platelets. The method is based on image segmentation so that we can easily separate WBCs from rest of the blood contents from the background and finally separation of nucleus and cytoplasm. As cytoplasm is insufficient so we have considered only the nucleus as the region of interest and its essential features are extracted.

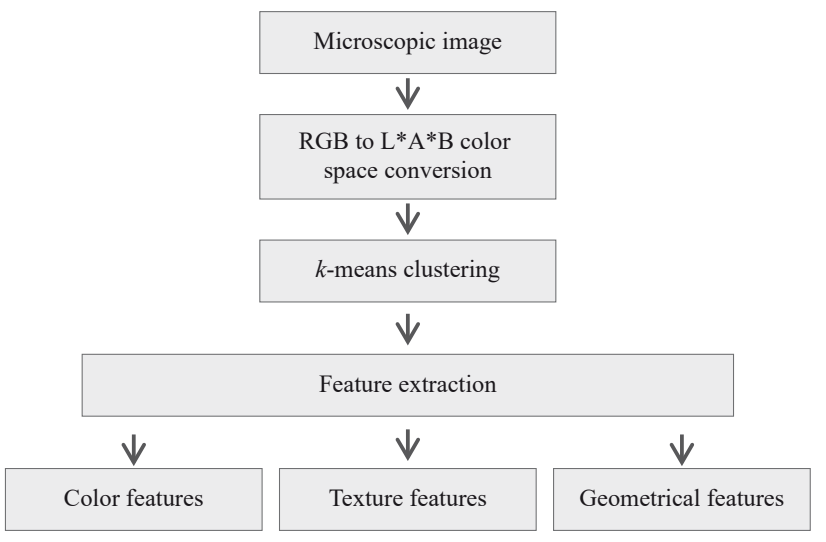

Fig. 1. Overview of the system. 


\section{The Database}

For the diagnosis of leukemia the visual analysis of peripheral blood samples is an important task. ALL-IDB i.e. the acute lymphoblastic leukemia image database for image processing is a public dataset of blood samples specifically designed for evaluation and the comparison of algorithm for segmentation and classification for each image in the dataset, the classification of cell is given and it is provided a specific set of figures of merits to be processed in order to fairly compare different algorithm when working with the proposed dataset. It contains about 39000 blood elements where the lymphocytes have been labeled by expert oncologists.

\section{Image Segmentation}

Image segmentation has drawn attention from many years in the field of research. There are many algorithms used in segmentation. So, algorithm that is developed for a particular set of images can't be used for another set of images.

The techniques used for image segmentation in this paper are $\mathrm{K}$ mean and clustering and Lab color space.

\section{A. $L * A * B$ Color Space:}

Typically an image can be represented with the help of three color components. Images generated by the digital microscopes are usually in RGB color space which is visually difficult to segment. For better color based segmentation we map the RGB image into $\mathrm{L}^{*} \mathrm{a}^{*} \mathrm{~b}^{*}$ color space. This color space consists of a luminosity layer $\mathrm{L}^{*}$, chromaticity layers $a^{*}$ and $b^{*}$. Since all the color information is in the $a^{*}$ and $b^{*}$ layers we use these two components for nucleus segmentation.

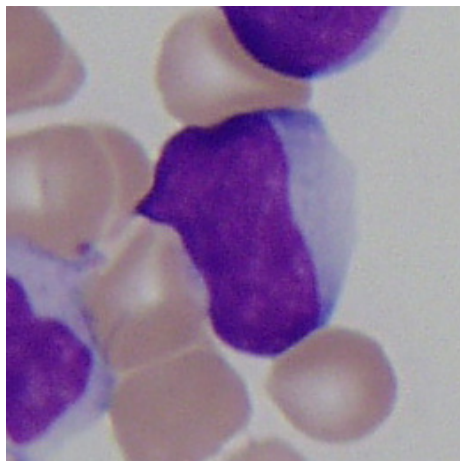

Fig. 2. Original Image.

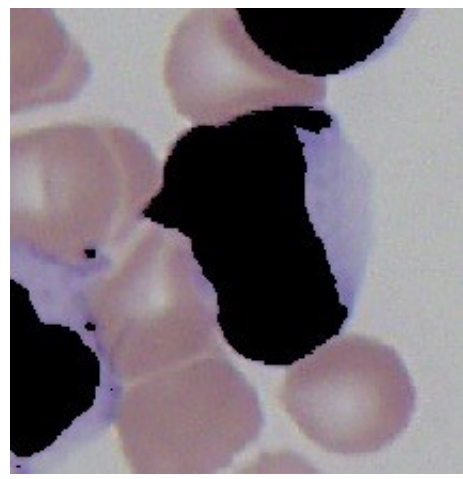

Fig. 3. Image after $\mathrm{L}^{*} \mathrm{~A} * \mathrm{~B}$.

\section{B. $K$-means Clustering:}

$k$-means clustering is a renowned subdividing method. In this substances are classified as belonging to one of k-groups. The consequences of partitioning method are a set of $\mathrm{K}$ clusters, each body of data set belonging to one cluster. In each cluster there may be a centroid or a cluster representative. In case where we reflect real-valued data, the arithmetic mean of the feature vectors for all objects within a cluster provides a suitable representative; alternative types of centroid may be mandatory in other cases. Clustering technique is used to create clusters from observations. It attempts to achieve partition such that objects within each cluster are as close to each other as possible, and as far from objects in other clusters as possible.

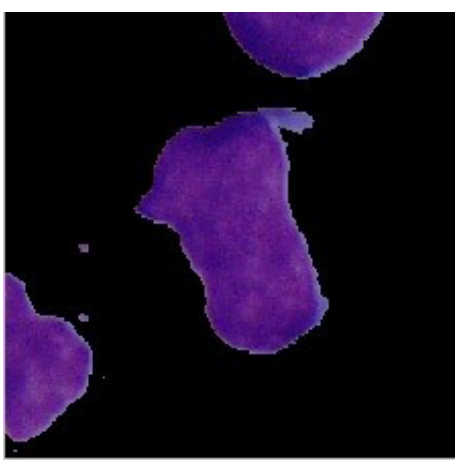

Fig. 4. Image after $k$ mean technique.

\section{FEATURE EXTRACTION}

Feature extraction in image processing is a system of redefining a great set of dismissed data, into a set of features of reduced dimension by transforming the input data into the set of features is called feature extraction. In feature extraction color, shape and geometrical features are observed for nucleus and the cell separately. The two clusters are made through k-mean, cluster 2 is for detecting the infected cells whereas cluster 3 is for nucleus. The basic inbuilt functions of feature extraction used in MATLAB are used.

\section{NeURAL Networks}

A neural network is an artificial system. In neural network the imitation of the activity of the brain is done. It imitates how the brain's neurons send messages through each other depending upon the work load some times. Several computing cells work in parallel to produce a result. This is usually seen as one of the possible ways artificial intelligence can work. Most neural networks can tolerate if one or more of the processing cells fail. A neural network is provided that has the capacity of "learning" to distinguish among patterns of data which may differ recognizably from idealized cases, and is able to perform pattern recognition faster while utilizing less memory and fewer clock cycles than neural networks implemented on sequential processors. This implementation is simpler and faster because of an inherent similarity between the flow of information in the brain and in data flow architecture. In this paper the comparison between two neural network techniques is done i.e. feedforward network and the radial basis function neural network (rbfnn).

\section{A. Feed Forward Network:}

Feed forward networks consist of a series of layers. The first layer has a connection from the network input. Each subsequent layer has a connection from the previous layer. The final layer produces the network's output. There are hidden layers in between the input and the output layer. This hidden layer contains the weight on each node. The more the weight is the more work the node will do Feed forward networks can be used for any kind of input to output mapping. A feed forward network with one hidden layer and enough neurons in the hidden layers, can fit any finite input-output mapping problem. Every pattern must have the same number of elements as the net has input nodes (excluding the bias unit), and every target the same number of elements as the net has output nodes. 
Experiment results by feedforward network: The experiment results in feedforward are basically dependent on the neural nodes we are using. The more the weightage on the nodes the more work load it will take to give the output. It was experimented through different nodes, initially 50 nodes then 80 and then 100 nodes were used on classes A and $\mathrm{B}$ which represents the cell and the nucleus cluster respectively. Using 100 neural nodes made the system slow and inefficient so it is not suitable to use 100 nodes. Result for 50 and 80 nodes is below:

TABLE I

EXPERIMENTS DONE THROUGH DifFERENT NeURONS

\begin{tabular}{|c|c|c|}
\hline Classes & Using 50 neurons & Using 80 neurons \\
\hline Class A & $92 \%$ & $94.7 \%$ \\
\hline Class B & $96 \%$ & $94.8 \%$ \\
\hline
\end{tabular}

\section{B. RBFNN:}

Radial Basis Function Neural Network is a neural network system of good performance. It is efficient in finding the exact nonlinear system's mapping till we have the enough image samples. This neural network system uses the radial basis functions as activation functions. So, it is not dependent on the weights of the neurons. The output of the network is basically a linear combination of radial basis functions of the inputs and neuron parameters. The basic MATLAB technique for RBFNN is implemented for the experiment. Using RBFNN we have $100 \%$ accuracy in detecting the leukemia infected cells and nucleus.

\section{CONCLUSion}

The experiments were conducted using two neural network techniques that is feedforward network and the radial basis function. These two techniques were employed on the ALL-IDB images whose features were extracted after they were segmented using L*a*b color space technique and $k$-mean clustering. According to the experiments conducted above RBFNN is more efficient as it consumes less time and gives the $100 \%$ accuracy result. So, it's better to use RBFNN instead of feedforward network to detect the leukemia in the proposed system.

\section{ACKNOWLEDGEMENT}

This study was supported by Graphic Era Hill University, Dehradun, India. We also appreciate the cooperation of ALL-IDB for providing the database.

\section{REFERENCES}

[1] Azhari, Ed-Edily Mohd, et al. "Tumor Detection in Medical Imaging: A Survey."International journal of Advanced Information Technology 4 (2014).

[2] Athira Krishnan, Sreekumar K. "A Survey on Image Segmentation and Feature Extraction Methods for Acute Myelogenous Leukemia Detection in Blood Microscopic Images."

[3] Jagadeesh, S., E. Nagabhooshanam, and S. Venkatachalam. "Image processing based approach to cancer cell prediction in blood samples.”ME\&HWDS Int. J. Technol. Eng. Sci 1.1 (2013).

[4] Banzi, Jamali Firmat, and Xue Zhaojun. "Detecting Morphological Nature of Cancerous Cell Using Image Processing Algorithms.” International Journal of Scientific and Research Publications: 11.

[5] Mohapatra, Subrajeet, Dipti Patra, and Sanghamitra Satpathy. "Unsupervised Blood Microscopic Image Segmentation and Leukemia Detection using Color based Clustering." International Journal of Computer Information Systems and Industrial Management Applications 4 (2012): 477-485.

[6] Mohapatra, Subrajeet, Dipti Patra, and Sanghamitra Satpathy. "Automated leukemia detection in blood microscopic images using statistical texture analysis." Proceedings of the 2011 International Conference on Communication, Computing \& Security. ACM, 2011.

[7] Li, Yujie, et al. "An improved detection algorithm based on morphology methods for blood cancer cells detection." Journal of Computational Information Systems 7.13 (2011): 4724-4731.

[8] Labati, Ruggero Donida, Vincenzo Piuri, and Fabio Scotti. "AllIDB: The acute lymphoblastic leukemia image database for image processing." Image processing (ICIP), 2011 18th IEEE international conference on. IEEE, 2011.

[9] Alix-Panabières, Catherine, Sabine Riethdorf, and Klaus Pantel. "Circulating tumor cells and bone marrow micrometastasis." Clinical Cancer Research14.16 (2008): 5013-5021.

[10] Pullarkat, Vinod A., et al. "Mast cell disease associated with acute myeloid leukemia: detection of a new c-kit mutation Asp816His." American journal of hematology 65.4 (2000): 307-309.

[11] Kraeft, Stine-Kathrein, et al. "Detection and analysis of cancer cells in blood and bone marrow using a rare event imaging system." Clinical cancer research6.2 (2000): 434-442.

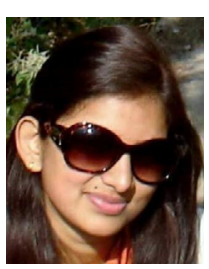

\section{Pragya Bagwari}

Pragya Bagwari is currently working as an Assistant Professor, department of CSE at IT Gopeshwar, Uttarakhand India. She has approx 2 years of Teaching and industrial experience. She done her B.Tech degree from UTU Dehradun and M.Tech degree from Graphic Era University Dehradun. Her area of Interest is Image processing, Wireless communication, Machine learning.

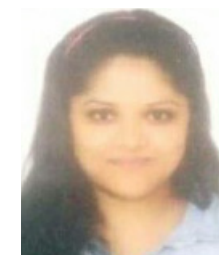

Bhavya Saxena

Bhavya Saxena is a research scholar. She obtained her B.Tech Degree from Rajasthan University and done her M.Tech from Graphic Era University. Her area of interest is Image processing and Wireless computing.

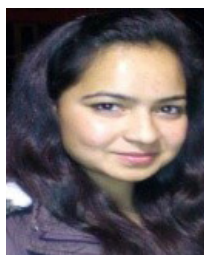

\section{Meenu Balodhi}

Meenu Balodhi obtained her Bachelors degree of Technology in Electronics \& Communication and Masters Degree (M.Tech) from Graphic Era University Dehradun. She was outstanding and meritious student during her degree program. She has 4 Years of Teaching Experince as an Assistant Professor in Uttaranchal University Dehradun. Her area of Interest is OFC, image Processing, Machine learning. She has Worked as PC member in several IEEE conferences and national conferences. She is the renowned author of many reputed research papers of national and international Journals.

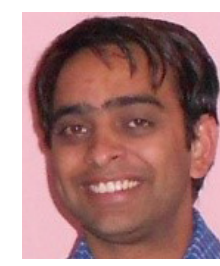

\section{Vishwanath bijalwan}

Vishwanath bijalwan obtained his B.Tech degree in ECE, from Dehradun Institute of Technology (UPTU) in 2009. He received his M.TECH degree from Uttarakhand Technical University in Digital Communication stream in 2012. Currently he is working as an Assistant Professor \& head of the department ECE at IT, Gopeshwar (State Government Institute). Besides this he has been also serving some more academic duties of Examination controller, \& Dean Student's welfare at IT Gopeshwar. He has total 5 years of teaching experience and 1 year industry experience. He has worked for various organizations, such as HCL Info system, Doon Institute of Engg. \& Technology Rishikesh, Uttaranchal University Dehradun, and Institute of Technology, Gopeshwar. In Research domain he has published various research papers in international journals. His research area of interest is Wireless adhoc network, IEEE 802.15.4, \& Machine learning. He has served as a PC member for many national \& International conferences in the region; he is also serving as a reviewing board member for various international journals like OMICS group and various IEEE conferences like ICACCCA, ICACCA, CICN, ICACCE. He is also an active member of IAENG, UACEE. His teaching area of Interest is Digital Electronics \& Design aspects, Electromagnetic field theory, Antenna \& wave propagation \& Digital signal processing. 